\section{Spreading the word}

\section{David Baulcombe and Roger Hull}

A SUBJECT that is attracting increasing attention among both plant virologists and cell biologists is the movement of viruses through plants. Progress in that area was evident at several recent meetings*, which have covered various aspects of the molecular biology of plant viruses. But the same meetings have demonstrated that molecular biology has confused further some of the thornier problems of plant virus taxonomy.

Viruses move through plants either by short-distance cell-to-cell spread through plasmodesmata or by long-distance spread through the vascular system. There is increasing evidence that cell-to-cell spread is facilitated by one or more virus gene products, the most studied of which is the P30 protein of tobacco mosaic virus. Using various manipulations of an infectious cDNA clone of the virus, W.O. Dawson and K. Lehto (University of California, Riverside) showed that the nearer a gene was to the $3^{\prime}$-end of the genome the more strongly it was expressed but that, within the limits examined, it was not the strength but the time of expression of P30 that was important for efficient virus spread; early expression was crucial for proper function. How P30 functions is still unknown but there are various pointers. The in vivo expressed product is phosphorylated (D. Zimmern and A. Haley, MRC Laboratory of Molecular Biology, Cambridge). Nicotinic acid blocks the transport of tobacco mosaic virus in leaf disks and this inhibition is overcome by cyclic AMP or by papaverin or calerotoxin, both of which increase cyclic AMP levels in leaves (J.G. Atabekov, Moscow State University).

\section{Movement models}

Broadly speaking, there are two models to explain how viruses move from cell to cell. In one, there is a specific interaction between the virus and the movement apparatus that allows the virus to translocate. In the alternative model, the intercellular connection is 'gated' so that abnormally large molecules, including virions or viral nucleoproteins, can pass through. So far, several lines of evidence point towards the second model, although there will be some complications. The absence of specificity in the process is suggested by the observations that some viruses assist movement of other viruses in certain hosts (Atabekov). In one example, this interaction is manifested as complementation of a defective homologue of $\mathrm{P} 30$ in tobacco rattle virus by intact * 5th International Congress of Virology, Kyoto, Japan, 20-27 August 1988; NATO Advanced Research Workshops, Chichester, UK, 12-16 April 1989 and York, UK, 2-7 July 1989; EMBO Workshop, Wye, UK, 16-19 July 1989. tobacco mosaic virus (V. Ziegler-Graff, Sainsbury Laboratory, Norwich).

Other experimental evidence for the gating model is provided by transgenic tobacco plants that express the P30 gene. In these plants the P30 protein is associated with plasmodesmata, especially in older leaves (D. Atkins, John Innes Institute, Norwich). The gating capacity of plasmodesmata in the plants was measured by video-intensified microscopy of fluorescent dyes of different sizes that had been encapsulated in liposomes and injected into the vacuole; the liposomes fused with the tonoplast membrane and released the dyes into the cytoplasm (W. Lucas, University of California, Davis). Control (non-transformed) plants did not allow cell-to-cell movement of dyes of relative molecular mass $\left(M_{\mathrm{r}}\right)$ greater than 800 , whereas in the transgenic plants the gating of $11,000-17,000 M_{\mathrm{r}}$ dyes was observed. Of course, viruses are much larger, so simple gating cannot be the whole story.

Although there is much less information about long-distance transport of viruses there is one important experiment that throws light on the involvement of the viral coat protein. T. Meshi and Y. Okada (University of Tokyo) mutated a cDNA clone of tobacco mosaic virus so as to disrupt the origin of virus assembly but to retain the coat protein sequence. This mutant, which expressed coat protein but was defective in particle assembly, spread systemically through the plant almost as rapidly as the wild-type virus. Thus, efficient assembly is not necessary for long-distance movement, though other experiments show that expression of coat protein is required.

A role of coat protein in transport of virus within the plant is also suggested from data obtained with plants transformed to express the viral coat protein. These plants are resistant to viral infection due to an action of the coat protein at several points in the infection cycle. An effect on transport is inferred from experiments in which efficient movement of virus through the stem is prevented by grafting a section of a plant expressing coat protein into the movement path of the virus (R. Beachy, Washington University, St Louis). Curiously, it was necessary to include a leaf on the grafted segment, otherwise the virus moved normally.

There is also an effect of coat-protein expression on virus disassembly, demonstrated in protoplasts of coat-proteinexpressing tobacco transfected with pseudovirions - non-viral RNA, in this case glucuronidase mRNA, coupled to viral origin-of-assembly and packaged in vitro (T.M.V. Wilson, John Innes Institute,
Norwich). Expression of pseudovirions was inhibited in mesophyll protoplasts but not in epidermal cells. As the epidermis is where infection is established, at least for mechanically-inoculated tobacco mosaic virus, it may be necessary to invoke another level of coat-protein action in order to reconcile the mesophyll specificity with the observation that coat-protein expression on a local lesion reduces the efficiency with which viral infection is established (Beachy). Thus, the analysis of coat-protein-mediated resistance is leading to discovery of new processes in the viral infection cycle. In addition, the complex mechanism underlying the resistance suggests that this will be an effective and durable trait for use in agriculture. The acid test will be in the field under a variety of conditions, and subject to environmental acceptability.

\section{Taxonomy}

Viral taxonomy has always been a difficult area and is likely to remain so following new discoveries about the organization of RNA viral genomes. Many of the previous groupings have now been blurred by the discovery that relationships between viruses vary in different parts of the genome. For example, the three different luteoviruses sequenced are all interrelated at their $3^{\prime}$-ends but at their $5^{\prime}$-ends the relationships differ. As summarized by R. Goldbach (Agricultural University, Wageningen), the $5^{\prime}$-end of beet western yellows luteovirus is similar to southern bean sobemeovirus; barley yellow dwarf luteovirus is similar to tobacco necrosis virus and carnation mottle virus; potato leafroll luteovirus has no obvious similarity to any of these viruses.

Another example of RNA recombination that may throw light on the mechanism of hybrid genome formation is the template switching observed in satellite RNAs of turnip crinkle tombusvirus. These RNA satellite molecules comprise, in part, a true satellite RNA that is not obviously similar to the helper virus and, in other parts, remnants of the helper virus genome. It appears that the replicase, having completed synthesis on one strand, or dropped off that strand, has a propensity to re-initiate synthesis at internal sites with similarity to the terminal promoters (A. Simon, University of Massachusetts). Transcription continues contiguously with the pre-existing RNA strand, producing a mosaic of genomic RNA sequences.

Clearly, good progress has been made with the analysis of the viral side of the pathogenic interaction; one looks forward to equally good progress with the analysis of the diseased and resistant plant.

David Baulcombe is in the Sainsbury Laboratory, Norwich NR4 7UH, and Roger Hull in the Department of Virus Research, John Innes Institute, Norwich NR4 7UH, UK. 